\title{
GEIDST
}

\section{CHLAMYDIA TRACHOMATIS NUMA CONSULTA DE INFECÇÕES SEXUALMENTE TRANSMISSíVEIS - ESTUDO RETROSPECTIVO DE QUATRO ANOS (2006-2009)}

Rodrigo Araúio Carvalho, Cândida Fernandes, Raquel Santos, Ana Rodrigues, Jorge Cardoso Serviço de Dermatologia e Venereologia, Hospital de Curry Cabral

RESUMO - A infecção genital por Chlamydia trachomatis $(\mathrm{Ct})$ é frequentemente assintomática e quando não tratada precocemente constitui um problema major de saúde pública. Os autores realizaram um estudo cujo objectivo foi determinar a prevalência de infecção genital por Ct numa consulta de Infecções Sexualmente Transmissíveis (ISTs) e realizar uma análise de diferentes variáveis. Para tal foi realizado um estudo retrospectivo dos doentes com diagnóstico de infecção por Ct confirmado por técnica de amplificação de ácidos nucleicos, observados na consulta de ISTs do Serviço de Dermatologia e Venereologia do Hospital Curry Cabral entre Janeiro de 2006 e Dezembro de 2009. Encontrou-se uma prevalência de infecção de 10,5\% (81 doentes com infecção genital por Ct em 771 indivíduos testados). Na população com infecção por Ct foram encontradas diferenças com significado estatístico entre o sexo masculino e feminino na idade média dos doentes (homens $=28,9$ vs mulheres $=24,3$ ) $p=0,018$; no número de companheiros sexuais nos últimos 6 meses (homens $=3,32$ vs mulheres $=1,50) p=0,009$; e no tempo em semanas decorrido entre $\circ$ início dos sintomas e o diagnóstico (homens $=2,65$ vs mulheres $=6,64) p=0,032$. Os doentes com co-infecção pelo Vírus da Imunodeficiência Humana $(\mathrm{VIH})$ tinham um maior número de companheiros sexuais nos últimos 6 meses do que os não $\mathrm{VIH} p=0,003$. Assim, existem diferenças demográficas, comportamentais e clínicas entre o sexo masculino e feminino e entre a população VIH e não VIH. Além disso, a elevada prevalência de infecção por Ct justifica os programas de diagnóstico precoce instituídos.

PALAVRAS-CHAVE - Chlamydia trachomatis; Infecção genital; Infecção sexualmente transmissível.

\section{CHLAMYDIA TRACHOMATIS IN A SEXUALLY TRANSMITTED DISEASE CLINIC - FOUR YEARS RETROSPECTIVE STUDY (2006- 2009)}

ABSTRACT - Chlamydia trachomatis $(\mathrm{Ct})$ is often responsible for an asymptomatic genital infection and represents a major public health problem when untreated. The authors objective was to determinate the Ct genital infection prevalence in a Sexual transmitted infections (STIS) clinic, and to perform an analysis of different variables. It was a retrospective study of all the patients with a Ct infection, observed in the STIs clinic of the Dermatology and Venereology department of Curry Cabral Hospital, between January 2006 and December 2009. The diagnosis was confirmed by Nucleic acid amplification technique. An infection prevalence of 10,5\% was found (81 patients with infection in 771 tested). In the 81 infected patients, statistic differences were found in the medium age between male and female patients (male $=28,9$ vs female $=24,3) p=0,018$; in the number of different sexual partners in the last 6 months (men $=3,32$ vs female $=1,5) p=0,009$; and in the time in weeks between the beginning of symptoms and the diagnosis (male $=2,65 \mathrm{vs}$ female $=6,64) p=0,032$. The patients with Human immunodeficiency virus (HIV) co-infection had a superior number of different sexual partners in the last 6 months comparing with the non HIV $p=0,003$. We concluded that in our patients there were demographic, behavioral and clinical differences between male and female, and between HIV and non HIV. Besides that, the elevated prevalence that was found reinforces the importance of the programs instituted for early diagnosis of Ct infection.

KEY-WORDS - Chlamydia trachomatis; Genital infection; Sexual transmited infection. 


\author{
Dr. Rodrigo Araújo Carvalho \\ Serviço de Dermatologia \\ Hospital de Curry Cabral \\ Rua da Beneficência 1069-166 Lisboa \\ Tel./Fax: 217924274 \\ E-mail: rodrigoaraujocarvalho@gmail.com
}

\section{INTRODUÇÃO}

A Chlamydia trachomatis (Ct) é a infecção de transmissão sexual (ISTs) bacteriana de maior prevalência no mundo ocidental estimando-se, segundo dados da Organização Mundial de Saúde, a ocorrência de 89 milhões de novos casos por ano em todo o mundo'. É responsável por diferentes entidades clínicas, sendo os serovars $A, B, B 1$ e $C$ associados ao tracoma, os serovars $D$ a $K$ a infecção genital, e os serovars $L 1$ a $L 3$ ao Linfogranuloma venereo (LGV). A infecção genital tem a particularidade de ser frequentemente assintomática e quando não tratada precocemente é uma das principais causas de infertilidade, gravidez ectópica e doença inflamatória pélvica, constituindo assim um problema major de saúde pública ${ }^{2}$. Por outro lado, à semelhança de outras ISTs, é co-factor da infecção pelo vírus da imunodeficiência humana (VIH), com o qual partilha vias de transmissão e factores de risco, aumentando entre duas a cinco vezes a probabilidade de transmissão deste vírus ${ }^{3}$.

Nos últimos anos, tem existido uma evolução significativa na sensibilidade e especificidade dos métodos utilizados para o diagnóstico laboratorial da infecção genital por $\mathrm{Ct}^{4}$, particularmente úteis nesta patologia em que uma abordagem exclusivamente semiológica é insuficiente para o seu diagnóstico, controlo e quebra da cadeia de transmissão ${ }^{5}$. Simultaneamente, existe na comunidade científica um aumento crescente das evidências que favorecem a realização da pesquisa de infecção genital por Ct em indivíduos assintomáticos ${ }^{5,6}$. No entanto, permanece por esclarecer se esta deve ser realizada de forma sistemática ou direccionada para populações específicas ${ }^{5,7}$. A morbilidade associada às ISTs, assim como o seu papel na transmissão do VIH são o motivo pelo qual recomendações europeias ${ }^{8}$ defendem que a todos os doentes que recorram a uma consulta de IST seja efectuada por rotina, entre outros, a pesquisa de infecção por $\mathrm{C}$.

Assim, com o presente estudo, os autores procu- raram em primeiro lugar determinar a prevalência de infecção genital por Ct numa consulta de ISTs na área da grande Lisboa. Procuraram ainda realizar uma análise descritiva retrospectiva de variáveis demográficas, comportamentais e clínicas dos doentes com infecção genital por $\mathrm{Ct}$ e pesquisar a ocorrência de eventuais diferenças estatisticamente significativas das variáveis estudadas entre o sexo masculino e feminino e entre a população VIH e não VIH.

\section{METODOLOGIA}

Para a realização do presente estudo retrospectivo, procedeu-se à consulta dos processos clínicos dos doentes observados na Consulta de ISTs do Serviço de Dermatologia do Hospital Curry Cabral entre Janeiro 2006 e Dezembro 2009. O diagnóstico de infecção por Ct foi confirmado em todos os casos, após pesquisa por técnica de amplificação de ácidos nucleicos realizada na urina, exsudado uretral, cervical ou anal (BD ProbeTec ${ }^{T M}$ ET).

Foram determinadas as prevalências de infecção por $\mathrm{Ct}$ e, para os doentes com infecção, foram ainda pesquisadas diferenças entre o sexo masculino e feminino e entre a população $\mathrm{VIH}$ e não $\mathrm{VIH}$.

Os dados demográficos, comportamentais e clínicos dos doentes com infecção por $\mathrm{Ct}$ que foram avaliados incluíram sexo, idade, orientação sexual, número de parceiros nos 6 meses anteriores ao diagnóstico, sintomatologia, tempo de evolução dos sintomas e existência de co-infecção por VIH ou por outra IST.

Os testes de Wilcoxon-Mann-Whitney e o de X2 foram utilizados averiguar o significado estatístico das diferenças encontradas entre o sexo masculino e feminino e entre a população $\mathrm{VIH}$ e não $\mathrm{VIH}$. Foram consideradas estatisticamente significativas, todas as comparações com $p$ inferior ao valor de significância de $5 \%$. O resultado destes testes estatísticos foi obtido com o recurso ao programa informático SPSS ${ }^{\circledR} 16.0$ para o Windows $X \mathrm{P}^{\circledR}$. 


\section{RESULTADOS}

Durante o período em causa foi realizada a pesquisa de infecção por Ct em 771 indivíduos, que foi diagnosticada em 81 doentes (10,5\%). Quando se procedeu ao cálculo da prevalência de infecção por $\mathrm{Ct}$ em diferentes grupos, constatou-se a existência de uma prevalência de $10,7 \%$ no sexo masculino $V s$ s $10 \%$ no sexo feminino; $18,2 \%$ na população com idade inferior a 25 anos $V s$ $8 \%$ na população com idade igual ou superior a 25 anos; $10,7 \%$ na população heterossexual $V s$ 10\% na população MSM; 7,4\% na população com 1 ou menos parceiros sexuais nos 6 meses anteriores $V s \quad 14,6 \%$ na população com mais de 1 parceiro sexual; $10,4 \%$ na população $\mathrm{VIH}$ negativa $\mathrm{Vs} 11,6 \%$ na população $\mathrm{VIH}$ positiva (Quadro I).

Dos 81 doentes com diagnóstico confirmado de Ct, 63 eram do sexo masculino e 18 do sexo feminino, com um ratio $M: F 3,5: 1$. A idade no média no sexo masculino era de 28,90 anos (mínima de 17 e máxima de 53 anos), significativamente superior à do sexo feminino que era de 24,33 anos (mínima de 14 e máxima de 52 anos), $p<0.05$. Em média, o número de parceiros sexuais nos 6 meses anteriores ao diagnóstico foi significativamente superior no sexo masculino $(3,32)$ do que no sexo feminino $(1,50), p<0.05$. Quando foram analisadas as variáveis clínicas, constatou-se que em ambos os sexos a maioria dos doentes apresentava sintomatologia, sem diferença estatisticamente significativa entre o sexo masculino e feminino, $p>0.05$. Assim, no sexo feminino documentava-se cervicite em $61 \%$ dos doentes $(n=11)$ enquanto que os restantes $39 \%(n=7)$ eram assintomáticos. No sexo masculino documentava.se uretrite em $76 \%$ dos doentes $(n=48)$, proctite em $6 \%(n=4)$ e $18 \%(n=11)$ eram assintomáticos. Quando existiam sintomas, o tempo médio de evolução foi significativamente superior no sexo feminino (6.64 semanas) do que no sexo masculino $(2,65$ semanas), $p<0.05$. No que respeita a outras ISTs concomitantes (não $\mathrm{VIH}$ ) na data do diagnóstico da infecção por $\mathrm{Ct}$, existiam em $61 \%$ dos casos $(\mathrm{n}=11)$ do sexo femino e em $46 \%$ dos casos $(n=29)$ do sexo masculino, $p>0.05$ (Quadro II). No total, a maioria destes casos correspondiam a infecção pelo Vírus do Papiloma Humano $(n=20)$ e Neisseria gonorrhoeae $(n=13)$. Coinfecções menos frequentemente encontradas incluíram Sífilis recente $(n=3)$, Vírus Herpes simplex tipo $2(n=3)$ e Vírus da Hepatite $B(n=1)$.

Dos 81 doentes com diagnóstico confirmado de Ct, $7 \%(n=6)$ tinham co-infecção pelo VIH. A idade no média na população VIH era de 32,33 anos (mínima de 20 e máxima de 47 anos), enquanto que na população não $\mathrm{VIH}$ era de 25,53 anos (mínima de 15 e máxima de 53 anos), $p>0.05$. Em média o número de parceiros sexuais nos 6 meses anteriores ao diagnóstico foi significativamente superior na população $\mathrm{VIH}(4,00)$ do que na população não $\mathrm{VIH}(2,83), \mathrm{p}<0.05$. Em ambos os grupos a maioria dos doentes apresentava sintomatologia, presente em $90 \%(n=4)$ da população $\mathrm{VIH}$ e em $69 \%(n=59)$ da população não $\mathrm{VIH}, p>0.05$. No que respeita a outras ISTs concomitantes (não VIH), existiam em $90 \%(n=4)$ dos doentes $\mathrm{VIH}$ e em $48 \%(n=36)$ dos doentes não VIH, p>0.05 (Quadro III).

\section{Quadro I}

\begin{tabular}{|c|c|c|c|}
\hline \multicolumn{2}{|c|}{ Grupo } & \multirow{2}{*}{$\begin{array}{c}N \\
61 / 570\end{array}$} & \multirow{2}{*}{$\begin{array}{c}\text { Prevalência (\%) } \\
\qquad 10,7\end{array}$} \\
\hline Seyo & Masculino & & \\
\hline החה & Feminino & $20 / 201$ & 10,0 \\
\hline \multirow{2}{*}{ Idade } & $<25$ anos & $34 / 187$ & 18,2 \\
\hline & $\geq 25$ anos & $47 / 584$ & 8,0 \\
\hline \multirow{2}{*}{ Orientação sexual } & Hetero & $68 / 661$ & 10,3 \\
\hline & MSM & $13 / 110$ & 11,8 \\
\hline \multirow{2}{*}{$\begin{array}{l}\text { No parceiros sexuais nos } \\
\text { últimos } 6 \mathrm{~m}\end{array}$} & $\leq 1$ & $32 / 435$ & 7,4 \\
\hline & $>1$ & $49 / 336$ & 14,6 \\
\hline \multirow{2}{*}{ Co-infecção VIH } & VIH - & $73 / 702$ & 10,4 \\
\hline & $\mathrm{VIH}+$ & $8 / 69$ & 11,6 \\
\hline
\end{tabular}




\section{Quadro II}

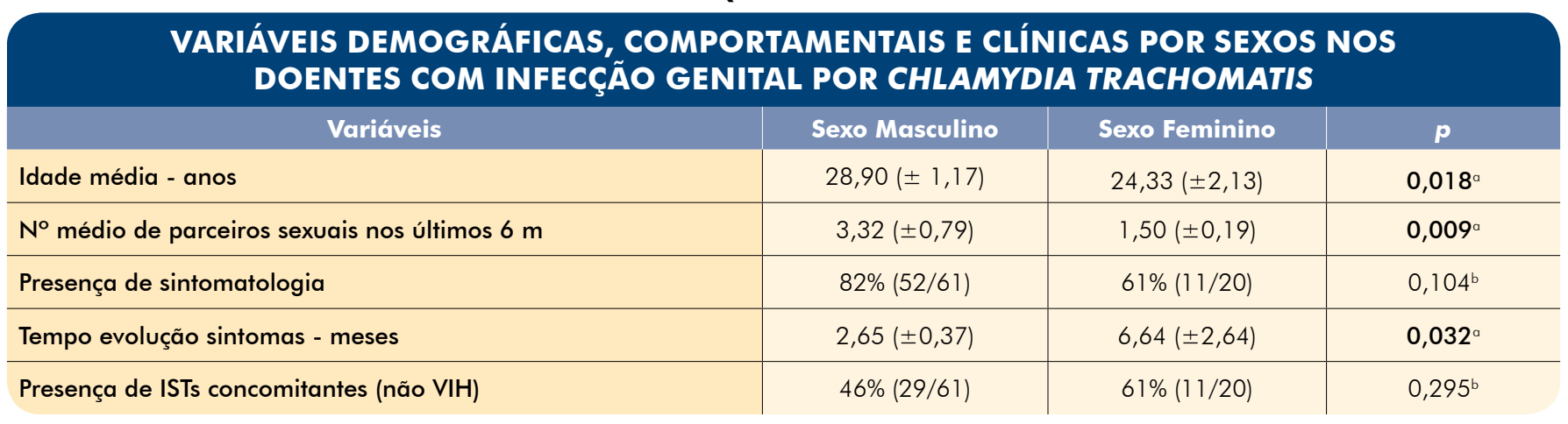

a - T. W. M. Whitney; b - T. X2

\section{Quadro III}

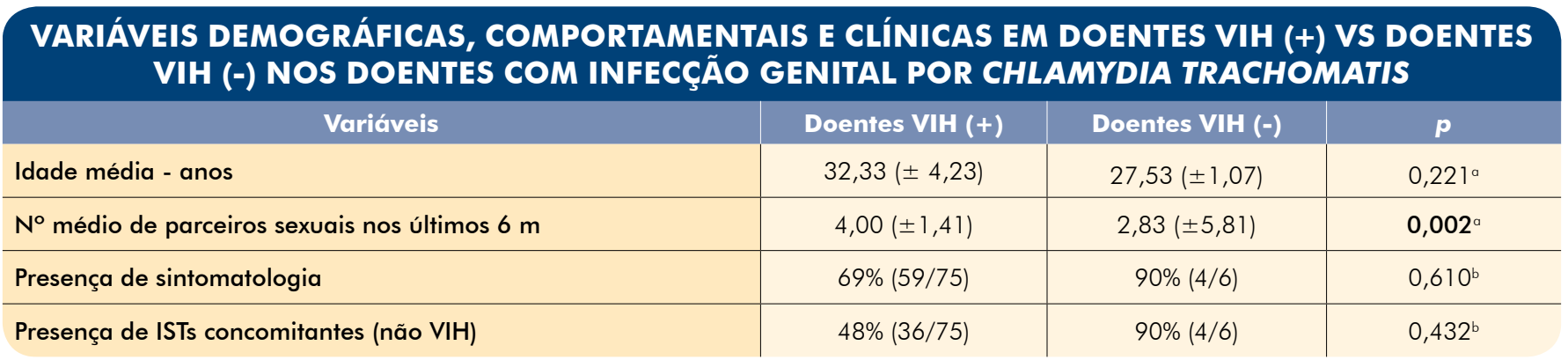

a - T. W. M. Whitney; b - T. x2

\section{DISCUSSÃO}

No presente estudo, encontrou-se uma prevalência global de infecção por $\mathrm{Ct}$ de 10,5\% (81/771). As limitações inerentes de uma amostra proveniente de doentes que recorreram a uma consulta de DSTs, não permitem a generalização dos dados obtidos para a população em geral. No entanto, existem na literatura publicados inúmeros estudos de prevalência de infecção por Ct, e alguns deles foram levados a cabo em populações com características sobreponíveis a esta. Cornelis A. ${ }^{9}$ e colaboradores encontraram uma prevalência base de infecção por $\mathrm{Ct}$ de 13,8\% (491/3568) num período de 30 meses numa consulta de DSTs em Denver, nos Estados Unidos da América. Já em território nacional, um estudo epidemiologico levado a cabo na consulta de DSTs do centro de Saúde da Lapa10, encontra uma prevalência de 7,9\% (173/2180) entre 2005 e 2006. Do mesmo modo, as prevalências encontradas no nosso estudo não oscilam muito quando comparadas entre sexos, orientação sexual e co-infecção com o VIH (Quadro I). Além disso, estes valores encontrados estão dentro do esperado quando comparados com estudos que analisam as mesmas variáveis. Assim, Benn $P D^{11} \mathrm{e}$ colaboradores reportam uma prevalência de infecção por Ct de $11 \%$ em 599 MSMs (no nosso estudo é de $11,8 \%$ - 13/110). Já em populações com VIH conhecido, Iwuji $C^{12}$ e colaboradores reportam $9 \%$ de infecção por $\mathrm{Ct}$ em 218 doentes $\mathrm{VIH}$, enquanto Srifeungfung $\mathrm{S}^{13}$ e colaboradores reportam $9,7 \%$ de infecção por $\mathrm{Ct}$ em 824 doentes $\mathrm{VIH}$ (no nosso estudo é de 11,6\% - 8/69).

A prevalência de infecção por $\mathrm{Ct}$ encontrada foi superior nos adultos jovens (18,2\% - 34/187). Assim, Borges da Costa $\mathrm{J}^{14}$ e colaboradores encontraram em 2007, numa clínica de ISTs em Lisboa, uma associação significativa entre infecção e mulheres jovens. Também Richey $\mathrm{C}^{15}$ e colaboradores encontraram uma prevalência mais elevada $(19,7 \%)$ nos indivíduos com idade inferior a 25 anos, dados estes que se repetem em diferentes publicações. A prevalência de infecção por Ct foi também mais elevada nos indivíduos com número recente superior de diferentes parceiros sexuais $(14,6 \%$ - 49/336), números estes que estão igualmente de acordo com o que tem sido publicado nos últimos anos ${ }^{16}$.

Dos 81 doentes com diagnóstico confirmado de Ct no presente estudo, constatou-se que no sexo masculino a idade média e o número de parceiros sexuais nos últimos 6 meses, eram significativamente superiores quando 
comparados com o sexo feminino. No entanto, esta diferença reflecte provavelmente aquela que é encontrada quando se avaliam as diferenças demográficas e comportamentais entre sexos dos utentes de uma consulta de DSTs ${ }^{17,18}$. Do mesmo modo, quando foram analisadas as variáveis clínicas, constatou-se que em ambos os sexos a maioria dos doentes apresentava sintomatologia, reflectindo provavelmente as características específicas desta população de utentes da consulta de DSTs, já que a infecção por C† na população em geral é caracterizada por ser na maioria das vezes assintomática.

Por fim, constatou-se que nos 81 doentes com diagnóstico confirmado de $\mathrm{Ct}$, o número de parceiros sexuais nos últimos 6 meses era significativamente superior na com população com co-infecção VIH. Assim, têm-se multiplicado relatos que constatam um aumento recente dos comportamentos de risco na população $\mathrm{VIH}$, quer por um fenómeno descrito na literatura anglo-saxónica como "prevention fatigue", que pode estar associado a um optimismo excessivo em relação à terapêutica antiretroviral e a uma menor preocupação na transmissão do vírus ${ }^{19-21}$.

\section{CONCLUSÃO}

A prevalência global de infecção por Ct encontrada na presente revisão está de acordo com aquilo que tem sido descrito em outros estudos levados a cabo em populações comparáveis. Ao mesmo tempo, também aqui se encontra uma elevada prevalência de infecção nos adultos jovens, o que parece justificar os programas instituídos de diagnóstico precoce.

Por outro lado, podemos afirmar que nos doentes com diagnóstico de infecção por $\mathrm{C} t$ do presente estudo, parecem existir algumas diferenças significativas do ponto de vista demográfico, comportamental e clínico entre o sexo masculino e feminino, que reflectem provavelmente as diferenças que existem na população que recorre a uma consulta de DSTs.

Por fim, o diagnóstico de infecção por Ct torna-se importante também na população $\mathrm{VIH}$, onde o aumento recente dos comportamentos de risco parece uma realidade.

\section{REFERÊNCIAS}

1. Gerbase A, Rowley J, HeymannD et al.: Global prevalence and incidence estimates of selected curable STDs Sex Transm Infect 74: S12-4 (1998).
2. Simms I: Epidemiology of chlamydia trachomatis. In International Handbook of Chlamydia. Moss T (ed.), UK, Alden Press, 2008, pp 2-7.

3. Fleming DT, Wasserheit J: A comprehensive review of the influence of traditional STD on HIV transmission and implications for HIV prevention. Sex Transm Infect 75: 3-17 (1999).

4. Puolakkainen $M$, Hiltunen-Back E, Reunala $T$ et al.: Comparison of performances of two commercially available tests, a PCR assay and a ligase chain reaction test, in detection of urogenital Chlamydia trachomatis infection. J Clin Microbiol 36: 1489-93 (1998).

5. Costa JB: Rastreio de infecções sexualmente transmissíveis em indivíduos assintomáticos. Trab Soc Port Dermatol Venereol 68: 117-21 (2010).

6. Kamwendo F, Forslin L, Bodin L, Danielsson D: Programmes to reduce pelvic inflammatory disease - the Swedish experience. Lancet 351 (Suppl III): 25-8 (1998).

7. Boag F, Kelly F: Screening for Chlamydia trachomatis. Br Med J 316: 1474 (1998).

8. Radcliffe K: European STD Guidelines. Int J STD \& AIDS 12(suppl 3): S2-102 (2001).

9. Cornelis $A$ et al.: Incidence and Repeat Infection Rates of Chlamydia trachomatis Among Male and Female Patients in an STD Clinic. Sex Transm Dis 29(2): 65-72 (2002).

10. Santo I, Azevedo J, Cardoso J: A consulta de doenças sexualmente transmissíveis do centro de saúde da Lapa. Caracterização da população utente e padrão epidemiológico das IST Trab Soc Port Dermatol Venereol 65(3): 365-74 (2007).

11. Benn PD, Rooney $G$ et al.: Chlamydia trachomatis and Neisseria gonorrhoeae infection and the sexual behaviour of men who have sex with men Sex Transm Infect 83: 106-12 (2007).

12. Iwuji CC, Reeves I, Nambiar K, Richardson D: Diagnostic utility of urethral smears in predicting urethral chlamydia in HIV-infected men. Int J STD AIDS 19(11): 741-3 (2008).

13. Srifeungfung $S$, Roongpisuthipong $A$ et al.: Prevalence of Chlamydia trachomatis and Neisseria gonorrhoeae in HIV-seropositive patients and gonococcal antimicrobial susceptibility: an update in Thailand. Jpn J Infect Dis 62(6): 467-70 (2009).

14. Borges da Costa J, Azevedo J, Santo I: Sexually transmitted infections and related sociodemographic factors in Lisbon's major Venereology Clinic: a descriptive study of the first 4 months of 2007. J Eur Acad Dermatol Venereol 24(7): $811-4$ (2010). 
15. Richey $C$, Macaluso $M$, Hook et al.: Determinants of reinfection with Chlamydia trachomatis. Sex Transm Dis 26: 4-1 1 (1999).

16. Sternberg $M$, Markowitz L et al.: Screening of males for Chlamydia trachomatis and Neisseria gonorrhoeae infections at STD clinics in three US cities Indianapolis, New Orleans, Seattle. Int J STD AIDS 15: 822 -8 (2004).

17. Hser YI: Cocaine use and high-risk sexual behavior among STD clinic patients. Sex Transm Dis 26: 82-6 (1999).

18. Benotsch E et al.: HIV Risk Behavior in Male and Female Russian Sexually Transmitted Disease Clinic Patients. Int J Behav Med 13: 26-33 (2006).
19. Dukers NHTM, Goudsmit J, de Wit JBF et al.: Sexual risk behaviour relates to the virological and immunological improvements during highly active antiretroviral therapy in HIV-1 infection. AIDS 15: 369-78 (2001).

20. Van de Ven P, Prestage G, Crawford J et al.: Sexual risk behaviour increases and is associated with HIV optimism among HIV-negative and HIV-positive gay men in Sydney over the four-year period AIDS 14: 2952-3 (2000).

21. Dodds JP, Nardone A, Mercey DE et al.: Increase in high risk sexual behaviour among homosexual men, London 1996-8: cross sectional, questionnaire study. BMJ 320: 1510-11 (2000). 